\title{
旅游干扰对非人灵长类动物的影响
}

\author{
范鹏来 ${ }^{1,2}$, 向左甫 ${ }^{2, *}$ \\ 1. 西南林业大学 云南省森林灾害预警与控制重点实验室, 云南 昆明 650224 ; \\ 2. 中南林业科技大学 生命科学与技术学院, 湖南 长沙 410004
}

摘要：随着经济发展及生活水平的提高, 以亲近自然为主要目的的生态旅游正成为公众的主要休闲方式。作为人类的 近亲, 非人灵长类动物在吸引游客方面具有天然的亲和力, 因此很多保护区及旅游景区正逐渐把 “猴园”、“猴谷” 及 “猴岛” 等作为招揽游客的平台。野外观猴能够提高公众的野生动物保护意识, 但同时也给动物带来了负面影响。该 文综述旅游活动对动物行为模式、繁殖和健康状况等的影响, 并对减少不利影响的措施进行归纳总结, 为开展非人灵 长类动物观赏旅游提供参考。

关键词: 非人灵长类; 旅游干扰; 旅游管理

中图分类号: Q959.848 文献标志码: A 文章编号: 0254-5853-(2013)01-0055-04

\section{Ecotourism disturbances to non-human primates}

\author{
Peng-Lai FAN ${ }^{1,2}$, Zuo-Fu XIANG ${ }^{2, *}$ \\ 1. Key Laboratory of Forest Disaster Warning and Control in Yunnan Province, Southwest Forestry University, Kunming 650224, China; \\ 2. College of Life Science and Technology, Central South University of Forestry \& Technology, Changsha 410004, China
}

\begin{abstract}
In tandem with economic growth and rising living conditions, ecotourism has increasingly gained popularity among the Chinese public. Non-human primates, as charismatic animals and the closest relatives of human beings, have shown a strong affinity in attracting the general public and raising money, and for that reason a variety of monkey parks, valleys, and islands are becoming increasingly popular in China. Though successful in raising a substantial sum of money for the managing agency of a nature reserve, there may be negative impacts on monkey groups used in ecotourism. Here, to establish effective guards for non-human primates involved in ecotourism, we present a review on tourism disturbance and summarize the negative impacts on behavioral patterns, reproduction, and health condition of animals.
\end{abstract}

Keywords: Non-human primates; Tourism disturbance; Tourism management

非人灵长类观赏旅游，不仅能够提高公众的自 然保护意识(Newsome et al, 2005; Fennell, 2008), 获 得稳定的资金以促进保护区和国家公园的基础设 施建设及物种保护，同时也可以提供给当地居民更 多的就业机会, 最终促进社区经济发展(Horwich, 1990; Brandon, 1996; Horwich, 1998; Zinner et al, 1998; Xiang et al, 2011)。然而, 与游客的长期接触 将不可避免地给动物带来一些负面影响。一般来 说, 对动物行为格局(Zhao, 1991; Treves \& Brandon, 2005; Hsu et al, 2009; Maréchal et al, 2011)、生理状
态(Behie et al, 2010; Maréchal et al, 2011)以及人兽 共患疾病传播(Wallis \& Lee 1999; Vié 1999; Lafferty et al，2002)等方面的干扰将影响种群健康和繁殖以 及种群结构和数量等(Jiang et al, 1994; Takahata et al, 1998; Torre et al, 2000; Berman et al, 2007)。但是, 在我国非人灵长类观赏旅游实践中, 很少有研究者 去证实这种负面影响，更少有管理者试图采取合理 措施去避免或者减少这种影响。本文着重从以上几 个方面介绍旅游对非人灵长类的负面影响, 期望引 起相关人员重视, 并为实施非人灵长类观赏旅游提

收稿日期: 2012-08-21；接受日期：2012-11-20

基金项目：国家自然基金 (31071937); 林业公益型项目 (201104073); 湖南省教育厅创新平台项目 (11K078)

“通信作者(Corresponding author), E-mail: zorph@126.com.

第一作者简介: 范鹏来 (1987-), 硕士研究生, 主要从事非人灵长类动物旅游干扰研究 
供参考。

\section{1 旅游对非人灵长类动物行为模式的影响}

一般来说, 旅游干扰会增加非人灵长类动物的 警戒时间、个体间争斗频率及移动距离, 甚至会改 变个体间等级关系等。因此, 动物的行为发生频次 经常被用作旅游干扰监测的指标之一。狝猴属动物 地理分布广泛, 种群数量大, 被普遍用于旅游参观。 Maréchal et al (2011) 发现雄性成年北非狝猴 (Macaca sylvanus)个体的自我擦拭(self-scratching) 行为与每天游客平均人数及游客与猴群之间的相 互作用(如参观、拍照、喂食、恐吓和攻击等)呈明 显正相关, 而与游客最高人数、观看时间百分比以 及观看距离无明显相关性。游客的存在会增加藏猕 猴(Macaca thibetana) 个体间的攻击行为, 并显著提 高婴猴死亡风险(Berman et al, 2007)。峨眉山藏猕猴 甚至会攻击为其投喂食物的游客(Zhao, 1991)。而接 受游客投食的台湾猕猴(Macaca cyclopis) 的攻击行 为是没有投喂食物猴群的四倍以上(Hsu et al, 2009)。

非人灵长类动物也会通过行为变化去应对旅 游干扰。例如, 在高强度旅游干扰条件下, 侏线猴 (Cebuella pygmaea) 会减少鸣叫, 呈现较少的社会玩 要行为和较低频率的攻击行为(Torre et al, 2000); 黑 吼猴(Alouatta pigra)会向较高的树冠移动(Treves \& Brandon, 2005); 红吼猴(Alouatta seniculus)会减少其 静坐观察的时间, 并将更多的时间用于取食和移动 活动的预算(Westin, 2007); 而大猩猩则会减少取 食时间并增加移动时间和距离等(Muyambi, 2005)。

\section{2 旅游对非人灵长类动物生理状态的影响}

物种长期处于应激状态时, 其垂体-下丘脑-肾 上腺(HPA)轴的反馈调节将促进肾上腺代谢激素的 分泌。作为物种压力应激反应的响应端, 肾上腺代 谢分泌的皮质醇 (cortisol) 和皮质酮(cortisone)可反 映物种压力水平。皮质醇为糖皮质激素, 由肾上腺 皮质最中层束状带分泌, 主要受 HPA 轴调节。非人 灵长类动物研究通常以皮质醇为目标激素(Francis et al, 1987; Sapolsky, 1993; Elder, 2001; Cross et al, 2004; Maréchal et al, 2011), 其粪便皮质醇浓度已被 广泛应用于个体急、慢性压力研究(Sapolsky, 1993; Crockett, 2000; Cross et al, 2004; Barbosa \& Mota, 2009; Maréchal et al, 2011)。

以非人灵长类动物作为旅游观赏动物的现象
在我国日益普遍，然而，目前国内还没有通过检测 内分泌水平对旅游干扰作出科学评估的报道。由于 检测技术和取样等多方面的原因, 直到 21 世纪对 野生非人灵长类动物内分泌的研究才较多地开展 起来。游客接触下的黑吼猴皮质醇含量高于对照种 群 (Behie et al，2010)。北非猕猴粪便皮质醇含量 与最大及平均游客人数、观看时间、以及观看距离 等因素明显相关，在游客的恐吓或攻击下，个体皮 质醇水平显著升高 (Maréchal et al，2011)。该现象 说明游客的存在可能给北非猕猴带来了焦虑, 而个 体只有当游客表现明显的攻击行为时才会出现强 烈的生理应激反应, 这可能是个体对于长期旅游干 扰所呈现的生理适应。婆罗洲猩猩 (Pongo pygmaeus morio)粪便的糖皮质激素水平在游客参 观后显著升高 (Muehlenbein et al, 2012)。然而, 在 同样的环境下，习惯化的个体糖皮质激素水平低于 同年龄未习惯化的个体。因此，非人灵长类旅游应 尽量选择习惯化时间较长的种群, 并适当限制观察 时间、距离及人数等。

\section{3 旅游对非人类灵长类动物繁殖的影响}

旅游干扰可能会影响非人灵长类的营养摄入 及繁殖。当前, 国内关于这部分的研究主要集中在 猕猴属。在海南南湾保护区, 以狝猴(Macaca mulatta) 作为旅游资源开发后，1985-1989 年，游客数量 年平均增长率为 $69.8 \%$, 猕猴的年平均繁殖率由 $77.8 \%$ 下降至 $70.5 \%$, 而新生猴的周平均死亡率则由 旅游开发前的零增加至 $2.4 \%$, 且在 1987 年间其周 平均死亡率甚至高达 $12.5 \%$ 。繁殖率降低的主要原 因是由于旅游参观人员拥挤逗弄, 甚至追打狝猴个 体, 造成其交配中断, 影响交配成功率甚至导致 孕猴流产死产(Jiang，1994)。旅游干扰对黄山藏狝 猴 (Macaca thibetana) 出生率没有影响, 但却明显增 加婴猴死亡风险 (Berman et al, 2007)。侏线猴 4 月 龄㚣猴存活率在低旅游压力区域为 $92 \%$, 而在高旅 游压力地区仅为 $60 \%$ (Torre et al, 2000)。然而, 游客 投食的日本猕猴(Macaca fuscuta) 群母婴出生率明 显高于野生群, 㚣猴死亡率则低于野生群, 而贞猴 性别比均接近 1:1 (Takahata et al, 1998)。

\section{4 旅游对于非人灵长类动物健康的影响}

疾病可能会在游客及野生动物之间传播。相似 的进化史使得病原体在人和非人灵长类动物的接 
触过程中更容易相互传播(Wallis \& Lee，1999)。 Muehlenbein et al (2010)的研究显示, 在接受调查的 633 名游客中, 有 $15 \%$ 的游客至少带有以下一种症 状: 咳嗽、喉咙痛、充血、发烧、腹江和区吐等。 调查显示近期与动物有近距离接触的参与者的呼 吸系统病征明显高于没有与动物接触的对照人群。 当前, 已经在多种非人灵长类动物中检测到人畜共 患的寄生虫。Vié (1999)在红吼猴中检测到对人类具 有较强致病性的雉虫和丝虫。Mbora \& McPeek (2009)的研究表明红疮猴 (Procolobus rufomitratus) 和白眉猴 (Cercocebus galeritus galeritus)的寄生虫 感染与人类活动相关。Teichroeb et al (2009)在黑白 病猴(Colobus polykomos)中检测到鞭虫和原虫, 并 且与人接触多的个体其粪便中的寄生虫数量也相 对较多。虽然一些重大疾病和动物死亡可能并非由 这些寄生虫所直接导致, 但寄生虫可降低个体受食 效率并影响其怀孕能力, 进而影响种群数量 (Gunn

\section{参考文献:}

Behie AM, Pavelka MS, Chapman CA. 2010. Sources of variation in fecal cortisol levels in Howler Monkeys in Belize. Am J Primatol, 72(7): 600-606.

Berman CM, Li JH, Ogawa H, Ionica C, Yin HB. 2007. Primate tourism, range restriction, and infant risk among Macaca thibetana at Mt. Huangshan, China. Int J Primatol, 28(5): 1123-1141.

Brandon KB. 1996. Ecotourism and Conservation: A Review of Key Issues. Washington D. C.: World Bank.

Chapman CA, Lambert JE. 2000. Habitat alteration and the conservation of African primates: case study of Kibale National Park, Uganda. Am J Primatol, 50(3): 169-185.

Cohen JJ, Crnic LS. 1983. Glucocorticoids, stress, and the immune response // Webb DR. Immunopharmacology and the Regulation of Leukocyte Function. New York: Dekker.

Crockett CM, Shimoji M, Bowden DM. 2000. Behavior, appetite, and urinary cortisol responses by adult female pigtailed macaques to cage size, cage level, room change, and ketamine sedation. Am J Primatol, 52(2): 63-80.

Cross N, Pines MK, Rogers LJ. 2004. Saliva sampling to assess cortisol levels in unrestrained common marmosets and the effect of behavioral stress. Am J Primatol, 62(2): 107-114.

Elder CM, Menzel CR. 2001. Dissociation of cortisol and behavior indicators of stress in an orangutan (Pongo pygmaeus) during a computerized task. Primates, 42(4): 345-357.

Fennell D. 2008. Ecotourism. 3rd ed. New York: Routledge.

Francis SJ, Walker RF, Riad-Fahmy D, Hughes D, Murphy JF, Gray OP. 1987. Assessment of adrenocortical activity in term newborn infants using salivary cortisol determinations. J Pediatr, 111(1): 129-133.
\& Irvine, 2003; Stokstad, 2004)。

总之，旅游干扰将直接影响非人灵长类动物在 自然状态下的某些特定生态或社会行为。例如，移 动距离增加会使得能量收支失去平衡, 进而导致体 力消耗过度体质变差; 性行为改变会引起交配失败 并导致繁殖率下降; 对后代照料行为的减少会影响 后代成活率; 长期处于胁迫环境亦会致使繁殖力及 免疫力下降, 且人猴共患疾病高发及出生率降低等 将最终影响种群健康及数量。因此, 在开展 “观猴” 生态旅游实践中要注意采取措施来避免或者减弱 这些不利影响, 例如, 建立专门的观猴通道, 禁止 人猴间零距离接触; 对游客参观猴群前进行紫外线 消毒; 严禁患有呼吸道疾病的人员接近猴群; 近距 离接近猴群的游客需使用医用口罩及防护性外套; 严格管理游客遗留的垃圾; 固定开放参观时间，及 时补充食物并要求管理人员采取严格措施预防传 染共患疾病传播等。

Gunn A, Irvine RJ. 2003. Subclinical parasitism and ruminant foraging strategies- a review. Wil Soc Bull, 31(1): 117-126.

Horwich RH. 1990. How to develop a community sanctuary an experimental approach to the conservation of private lands. Oryx, 24: 95-102.

Horwich RH. 1998. Effective solutions for howler conservation. Int $J$ Primatol, 19(3): 579-598.

Hsu MJ, Kao CC, Agormoorthy G. 2009. Interactions between Visitors and Formosan Macaques (Macaca cyclopis) at Shou-Shan Nature Park, Taiwan. Am J Primatol, 71(3): 214-222.

Jiang HS, Wang J, Liu ZH. 1994. Impact of tourism on macaca's population growth in Nanwang. Acta Ther Sin, 14(3): 166-171. [江海声, 王俊, 刘振 河. 1994. 旅游对南湾猕猴种群增长的影响. 兽类学报, 14(3): 166-171.]

Lafferty K D, Gerber L R. 2002. Good medicine for conservation biology: the intersection of epidemiology and conservation theory. Conserv Biol, 16(3): 593-604.

Lott DF, McCoy M. 1995. Asian Rhinos, Rhinoceros unicornis, on the Run? Impact of tourist visits on one population. Biol Conserv, 73(1): 23-26.

Mann J, Smuts B. 1999. Behavioral development in wild bottlenose dolphin newborns (Tursiops sp.). Behaviour, 136(5): 529-566.

Maréchal L, Semple S, Majolo B, Qarro M, Heistermann M, MacLarnon A. 2011. Impacts of tourism on anxiety and physiological stress levels in wild male Barbary macaques. Biol Conserv, 144(9): 2188-2193.

Mbora DM and McPeek MA. 2009. Host density and human activities mediate increased parasite prevalence and richness in primates threatened by habitat loss and fragmentation. J Ani Ecol, 78(1): 210-218.

Muehlenbein M P, Ancrenaz M, Sakong R, Ambu L, Prall S, Fuller G, 
Raghanti M A. 2012. Ape conservation physiology: Fecal glucocorticoid responses in wild pongo pygmaeus morio following Human Visitation. PLoS ONE, 7(3): e33357.

Muehlenbein MP, Martinez LA, Lemke AA, Ambu L, Nathan S, Alsisto S, Sakong R. 2010. Unhealthy travelers present challenges to sustainable primate ecotourism. Travel Med Inf Disease, 8(3): 169-175.

Muyambi F. 2005. The impact of tourism on the behaviour of mountain gorillas. Gorilla J, 30: 14-15.

Newsome D, Dowling RK, Moore SA. 2005. Wildlife Tourism. Clevedon: Channel View Publications.

Sapolsky RM. 1993. Potential behavioral modification of glucocorticoid damage to the hippocampus. Behav Brain Res, 57(2): 175-182.

Stokstad E. 2004. Forest loss makes monkeys sick. Science 305: 1230-1231. 2004. Loss of dung beetles puts ecosystems in deep doo-doo. Science, 305(5688): 1230.

Strier KB. 2007. Conservation // Campbell CJ, Fuentes A, MacKinnon KC, Panger M, Bearder SK. Primates in Perspective. New York: Oxford University Press, 496-509.

Takahata Y, Suzuki S, Agetsuma N, Okayasu N, Sugiura H, Takahashi H, Yamagiwa J, Izawa K, Furuichi T, Hill DA, Maruhashi T, Saito C, Sato S, Sprague DS. 1998. Reproduction of Wild Japanese Macaque Females of Yakushima and Kinkazan Islands: A Preliminary Report. Primates, 39(3): 339-349.

Teichroeb JA, Kutz SJ, Parkar U, Thompson RA. 2009. Pascale Sicotte1 Ecology of the Gastrointestinal Parasites of Colobus vellerosus at
Boabeng-Fiema, Ghana: Possible Anthropozoonotic Transmission. Am J Phys Anthropol, 140(3): 498-507.

Torre SD, Snowdon CT, Bejarano M. 2000. Effects of human activities on wild pygmy marmosets in Ecuadorian Amazonia. Biol Conserv, 94(2): 153-163.

Treves A, Brandon K. 2005. Tourism impacts on the behaviour of black howler monkeys (Alouatta pigra) at Lamanai, Belize // Paterson JD, Wallis J. Commensalism and Conflict: The Human-Primate Interface. Norman OK: American Society of Primatologists Publication, 146-167.

Vié JC. 1999. Wildlife rescues-the case of the Pet hydroelectric dam in French Guiana. Oryx, 33: 115-126.

Wallis J, Lee DR. 1999. Primate conservation: the prevention of disease transmission. Int J Primatol, 20(6): 803-826.

Westin JL. 2007. Effects of Tourism on the Behavior and Health of Red Howler Monkeys (Alouatta Seniculus) in Suriname. Dissertation, University of Michigan: 173 .

Xiang ZF, Yu Y, Yang M, Yang JY, Niao MY, Li M. 2011. Does flagship species tourism benefit conservation? A case study of the golden snub-nosed monkey in Shennongjia National Nature Reserve. Chn Sci Bull, 56(24): 2553-2558.

Zhao QK. 1991. Macaques and tourists at Mt. Emei, China. Res Expl, 7(1): 115-116.

Zinner D, Butynski TM. 1998. Status and conservation of African primates. Folia Primatol, 69(4): 193-197. 